\title{
Enhanced Human Gingival Fibroblast Response and Reduced Porphyromonas gingivalis Adhesion with Titania Nanotubes
}

\author{
Zhiqiang Xu $\left(\mathbb{D},{ }^{1,2}\right.$ Yuqi He, ${ }^{1}$ Xiufeng Zeng, ${ }^{3}$ Xiuxia Zeng, ${ }^{4}$ Junhui Huang, ${ }^{1}$ Xi Lin, ${ }^{5}$ \\ and Jiang Chen $\mathbb{1}^{2}$ \\ ${ }^{1}$ Department of Stomatology, Affiliated Hospital of Putian University, Putian, Fujian 351100, China \\ ${ }^{2}$ Department of Oral Implantology, Affiliated Stomatological Hospital of Fujian Medical University, Fuzhou, Fujian 350002, China \\ ${ }^{3}$ Hanjiang Zeng Xiufeng Dental Clinic, Putian, Fujian 351117, China \\ ${ }^{4}$ Department of Stomatology, Putian Hanjiang Hospital, Putian, Fujian 351100, China \\ ${ }^{5}$ Laboratory of Electron Microscopy, Public Technology Service Center, Fujian Medical University, Fuzhou, Fujian 350002, China
}

Correspondence should be addressed to Jiang Chen; dentistjiangchen@sina.com

Received 31 January 2020; Revised 24 April 2020; Accepted 23 May 2020; Published 8 June 2020

Academic Editor: Roya Dastjerdi

Copyright (C) 2020 Zhiqiang Xu et al. This is an open access article distributed under the Creative Commons Attribution License, which permits unrestricted use, distribution, and reproduction in any medium, provided the original work is properly cited.

\begin{abstract}
Successful dental implants rely on stable osseointegration and soft-tissue integration. Titania nanotubes (TNTs) with a diameter of $100 \mathrm{~nm}$ could increase the mesenchymal stem cell response and simultaneously decrease Staphylococcus aureus adhesion. However, the interactions between the modified surface and surrounding soft tissues are still unknown. In the present study, we fully investigated the biological behavior of human gingival fibroblasts (HGFs) and the adhesion of Porphyromonas gingivalis ( $P$. gingivalis). TNTs were synthesized on titanium (Ti) surfaces by electrochemical anodization at 10,30 , and $60 \mathrm{~V}$, and the products were denoted as NT10, NT30, and NT60, respectively. NT10 (diameter: $30 \mathrm{~nm}$ ) and NT30 (diameter: $100 \mathrm{~nm}$ ) could enhance the HGF functions, such as cell attachment and proliferation and extracellular matrix- (ECM-) related gene expressions, with the latter showing higher enhancement. NT60 (diameter: $200 \mathrm{~nm}$ ) clearly impaired cell adhesion and proliferation and ECM-related gene expressions. Bacterial adhesion on the TNTs decreased and reached the lowest value on NT30. Therefore, NT30 without pharmaceuticals can be used to substantially enhance the HGF response and reduce $P$. gingivalis adhesion to the utmost, thus demonstrating significant potential in the transgingival part of dental implants.
\end{abstract}

\section{Introduction}

Titanium (Ti) implants are widely used in dentistry to replace missing teeth due to their excellent material properties and biological activity $[1,2]$. However, given the exposure of the transgingival part to the oral environment, $\mathrm{Ti}$ implants are easily invaded by bacteria at implant-soft tissue sites. Bioactive molecules and antibacterial agents are introduced into the transgingival part to improve the soft-tissue integration and antibacterial activity $[3,4]$. However, these coatings are expensive and difficult to fabricate and also unsuitable for conventional implants with cylindrical or tapered shapes $[5,6]$. Therefore, the application of the transgingival part of dental implants with excellent soft-tissue integration and antibacterial property without pharmaceuticals is desirable.

Given the nanoscale dimensions of the physiological extracellular matrix (ECM), titania nanotubes (TNTs) manufactured on the Ti surface for the transgingival part of dental implants have received considerable attention $[4,7]$. As the major cells in peri-implant soft tissue, human gingival fibroblasts (HGFs) can secrete ECM containing abundant collagen fibers, which contribute to gingival wound healing repair and regeneration [4]. Therefore, the investigation of 
TABLe 1: Primers used for qRT-PCR.

\begin{tabular}{lcc}
\hline Gene & Forward primer sequence $\left(5^{\prime}-3^{\prime}\right)$ & Reverse primer sequence $\left(5^{\prime}-3^{\prime}\right)$ \\
\hline ITGB1 & GAATGCCTACTTCTGCACGAT & TTCCTTTGCTACGGTTGGTTA \\
VCL & CTCGTCCGGGTTGGAAAAGAG & AGTAAGGGTCTGACTGAAGCAT \\
FN & AGGAAGCCGAGGTTTTAACTG & AGGACGCTCATAAGTGTCACC \\
COLI & CGAAGACATCCCACCAATCA & GATCACGTCATCGCACAACA \\
GAPDH & TGGGTGTGAACCATGAGAAGT & TGAGTCCTTCCACGATACCAA \\
\hline
\end{tabular}
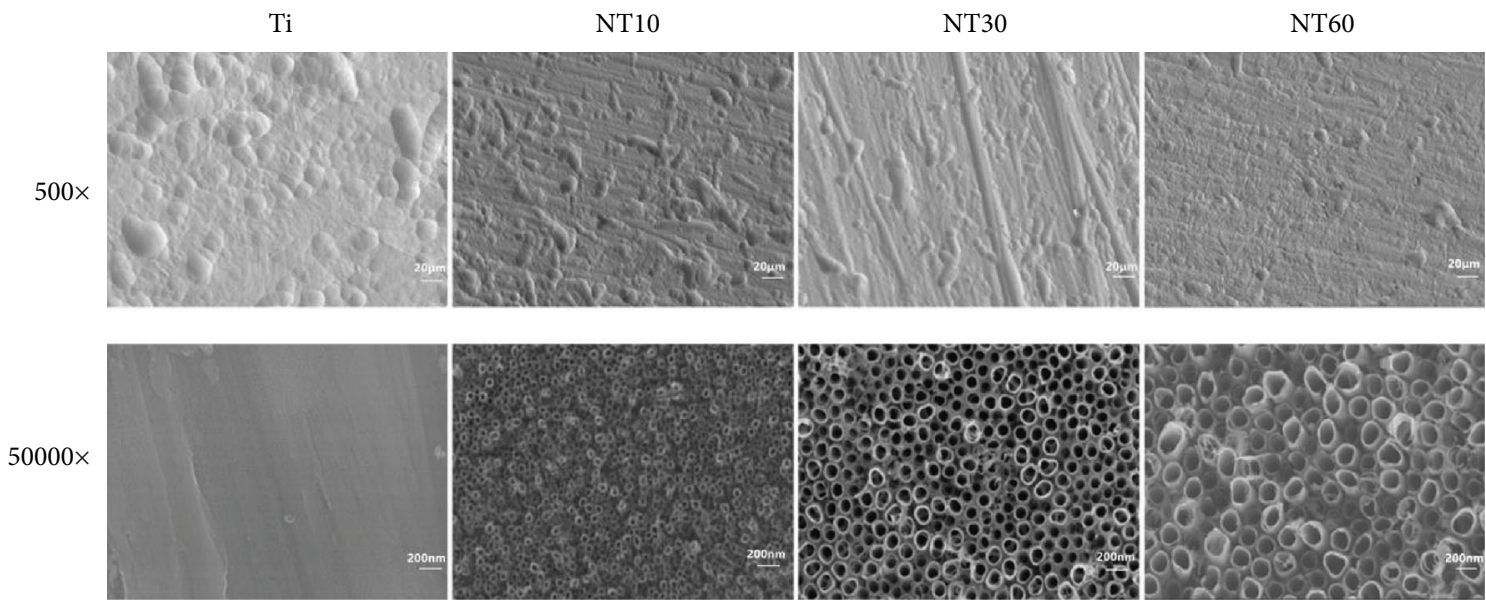

FIGURE 1: SEM images of different samples with two different magnifications of 500x and 50,000x [13].

the biological response of HGFs toward TNTs while assessing the interactions between TNTs and soft tissues was proposed. TNTs with a diameter of 70-90 nm have been reported to promote the cell adhesion and proliferation of HGFs and ECM-related gene expressions $[3,8]$. However, TNTs with a diameter of 50-100 nm have also been demonstrated to support the adhesion and proliferation of HGFs but without notable promotion $[9,10]$. TNTs with diameters of $15-$ $100 \mathrm{~nm}$ even seriously inhibited the adhesion and spread of HGFs, and the inhibitory effect increased with the increased diameter of TNTs [11]. In addition, $100 \mathrm{~nm}$ should not be the diameter threshold of TNTs to exhibit effects on the cell behavior. However, TNTs with a diameter of $>100 \mathrm{~nm}$ are difficult to prepare using traditional strong acid electrolytes.

Bacteria invade the implant surface during surgical operation and wound healing; thus, "the race for the surface" starts before soft-tissue integration occurs [12]. Thus, the potential antibacterial properties of dental implants are critical given the abundance of pathogens in the oral environment and inherent nonantibacterial nature of Ti. We have previously shown that TNTs could decrease the adhesion of Gram-positive Staphylococcus aureus even without drugs, which could present harmful side effects [13]. However, whether the result can be applied to other kinds of bacteria, such as the Gram-negative bacterium Porphyromonas gingivalis ( $P$. gingivalis, one of the most common bacteria in peri-implantitis), is still unknown. TNTs alone also been demonstrated to increase both the adhesion of Grampositive and that of Gram-negative bacteria, and this action might be due to the differences in their physical and chemical

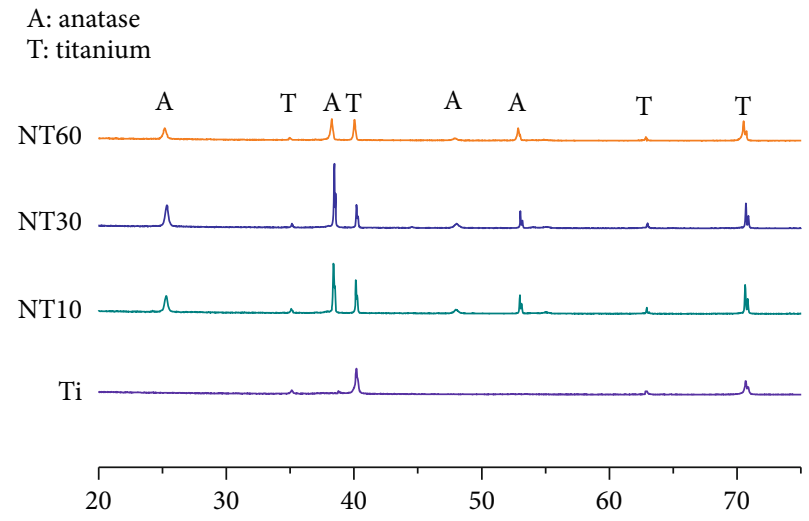

FIgURE 2: XRD patterns of TNTs after heat treatment at $450^{\circ} \mathrm{C}$ for $2 \mathrm{~h}$ and control Ti before heat treatment.

features [3]. Thus, the same TNTs for both HGFs and P. gingivalis should be assessed given the conflicting antibacterial assays.

Here, we aimed to manufacture optimum TNTs that enhance HGF response and simultaneously reduce $P$. gingivalis adhesion without pharmacological therapy. For this purpose, TNTs with three different diameters were manufactured using a weak acidic electrolyte, which can generate smoother and larger nanotubular layers within a suitable range of voltage compared with a strong acid electrolyte. For the first time, the response of HGFs and the adhesion of $P$. gingivalis on the three TNTs were systematically and simultaneously studied. 

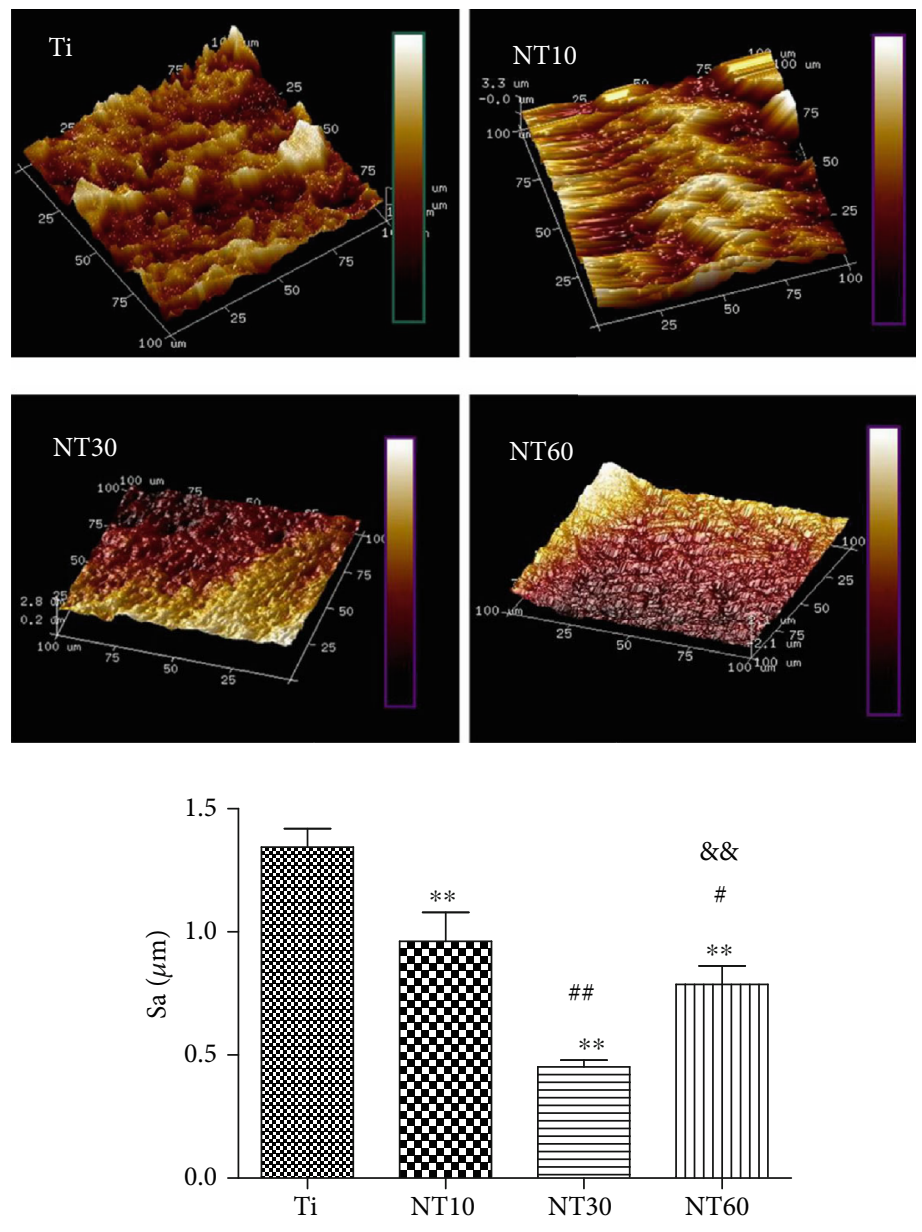

FIGURE 3: AFM images of different samples and multiple comparison results of surface roughness (Sa) on different samples.

\section{Materials and Methods}

2.1. Preparation of TNTs on Ti. TNTs were prepared via anodization on a Ti sheet. A $1 \times 1 \mathrm{~cm}^{2}$ Ti sheet was polished with a gradient of sandpapers in the same direction to create a grooved microrough surface and degreased by sonication in acetone and deionized water for $10 \mathrm{~min}$. The sheet was then eroded in $4 \mathrm{wt} \% \mathrm{HF}-5 \mathrm{~mol} / \mathrm{L}_{\mathrm{HNO}}$, rinsed with distilled water, and dried in air. As previously described, anodization was conducted using a mixture of $0.50 \mathrm{wt} \% \mathrm{NH}_{4} \mathrm{~F}+10 \mathrm{vol} \%$ $\mathrm{H}_{2} \mathrm{O}$ in glycerol at 10,30 , and $60 \mathrm{~V}$ (the samples were denoted as NT10, NT30, and NT60, respectively) for $3 \mathrm{~h}$ and ultimately annealed in air for $2 \mathrm{~h}$ at $450^{\circ} \mathrm{C}$ [13]. An identically sized $\mathrm{Ti}$ sheet with a native $\mathrm{TiO}_{2}$ oxide layer was used as the control. All samples were sterilized using ultraviolet irradiation $(0.5 \mathrm{~h}$ for either side) before use.

\subsection{Specimen Characterization}

2.2.1. Scanning Electron Microscopy (SEM). The surface morphology of the samples was observed by SEM. All samples were sputter-coated with platinum before observation.

2.2.2. Phase Analysis. The crystalline phase analysis of the samples was detected using X-ray diffraction (XRD).
2.2.3. Atomic Force Microscopy (AFM). Surface feature and roughness were examined with AFM in air tapping mode, with a scan size of $100 \mu \mathrm{m}$.

2.2.4. Contact Angle Determination. A $1 \mu \mathrm{L}$ sessile droplet of double distilled water was delivered to the specimen surface. Contact angles were measured using a drop shape analysis system.

2.2.5. Total Proteins and Fibronectin (FN) Adsorption Assay. A $1 \mathrm{~mL}$ droplet of Dulbecco's modified Eagle's medium-low glucose containing $10 \%$ fetal bovine serum (FBS) was pipetted onto each specimen in 24 -well plates. All specimens were transferred to new 24-well plates and gently rinsed with phosphate-buffered saline (PBS) after $2 \mathrm{~h}$. The total proteins adsorbed onto the samples were determined using a Micro BCA protein assay kit. The FN adsorbed onto the samples was determined using an enzyme-linked immunosorbent assay kit for FN.

2.3. Cell Cultures. HGFs were obtained from the Key Laboratory of Stomatology, Fujian Medical University. As previously described, the cells were obtained from healthy gingival tissues of orthodontic patients who had their premolar teeth removed [14]. Tissues from different donors were 
minced to approximately $1 \times 1 \mathrm{~mm}^{2}$ and then maintained in Dulbecco's modified Eagle's medium-low glucose supplemented with $10 \%$ FBS and $1 \%$ penicillin streptomycin. HGFs were digested and subcultured at a $1: 3$ ratio after reaching $80 \%$ confluence. Cells between passage 2 and passage 5 were used for the experiments. HGFs were seeded onto each sample in 24-well plates at a density of $4 \times 10^{4} /$ well for the cell adhesion assay and $2 \times 10^{4} /$ well for the other assays.

2.3.1. Cell Morphology. After culturing for 2 days, all the samples were fixed with $2.5 \%$ glutaraldehyde overnight. The samples were dehydrated in graded ethanol series and freezedried. All samples were sputter-coated with platinum before SEM observation.

2.3.2. Adhesion and Proliferation. For the cell adhesion assay, the cell nuclei were stained with 4,6-diamidino-2-phenylindole (DAPI) after $2 \mathrm{~h}$ of incubation and then observed under a fluorescence microscope. For the cell proliferation assay, the cells were evaluated using Cell Counting Kit-8 (CCK-8) assay after 1,3, and 7 days of incubation.

2.3.3. Gene Expressions. The relative gene expression levels of HGFs, including integrin $\beta 1$ (ITGB1, an important receptor mediating the cellular binding onto the implant surface) [15], FN (an important adhesion protein) [16], vinculin (VCL, an important cytoskeleton binding protein associated with adhesion strength and cell migration) [17], and type I collagen (COL1, the main component in gingival tissues) $[18,19]$, were measured using quantitative reverse transcription polymerase chain reaction (qRT-PCR). The total RNA was isolated from the cells by using Trizol reagent after being cultured for 7 days, and the CDNA was synthesized from the total RNA by using a cDNA Reverse Transcription Kit in accordance with the manufacturer's instructions. The qRTPCR analysis was conducted on an ABI Prism 7500 realtime PCR cycler with SYBR Premix Ex Taq ${ }^{\mathrm{TM}}$ II. Table 1 shows the primer sequences of the genes. The mRNA levels for cells were normalized for glyceraldehyde-3-phosphate dehydrogenase (GAPDH) mRNA.

2.4. Bacterial Culture. P. gingivalis (ATCC33277) was cultivated in brain heart infusion broth with $5 \%$ yeast extract, $1 \%$ hemin, and $0.2 \%$ vitamin $\mathrm{K} 1$ at $37^{\circ} \mathrm{C}$ under standard anaerobic conditions. The TNTs and control Ti were all separately placed in 24-well plates and incubated in $1 \mathrm{~mL}$ bacteria-containing medium $\left(10^{6} \mathrm{CFU} / \mathrm{mL}\right)$.

2.4.1. Bacterial Morphology. All samples were fixed with $2.5 \%$ glutaraldehyde for $4 \mathrm{~h}$, dehydrated in graded ethanol series, and sputter-coated with platinum for SEM observation.

2.4.2. Antibacterial Assay. In vitro antibacterial activity was assessed by a plate-counting method after culturing for $6 \mathrm{~h}$. The sample was ultrasonically agitated to detach the bacteria from the sample, and the viable bacteria in PBS were recultivated on agar plates for colony counting through 3 days of anaerobic incubation at $37^{\circ} \mathrm{C}$.
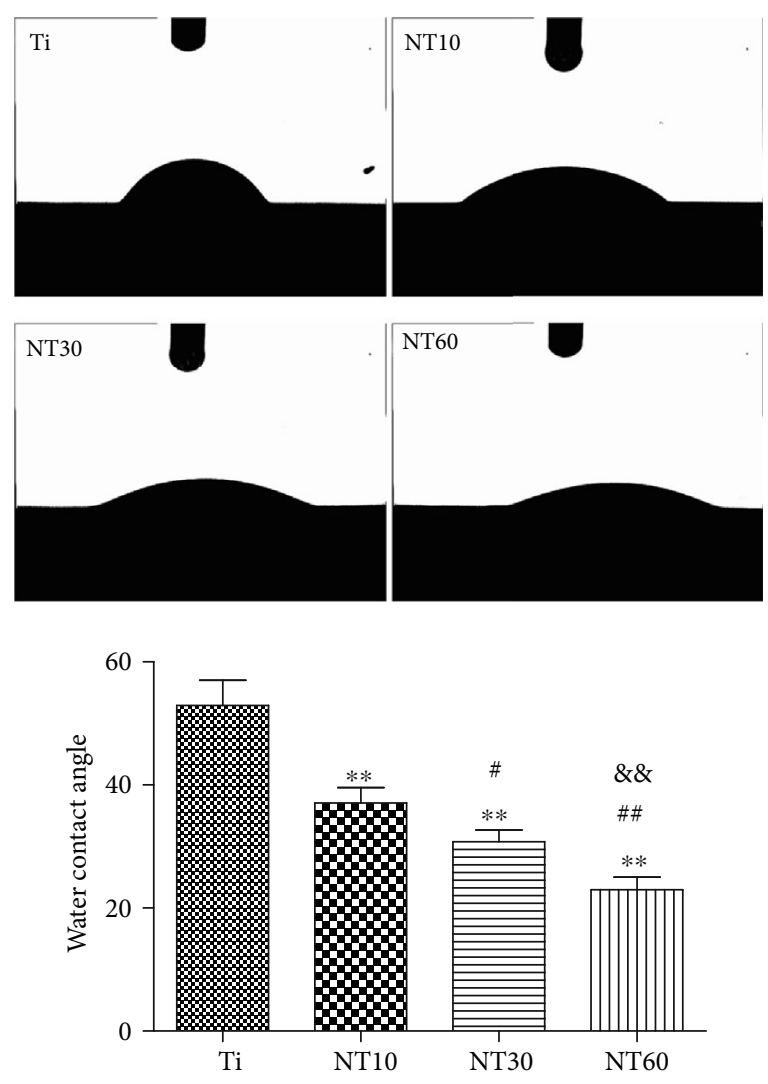

FIgURE 4: Images of water droplets on different samples and multiple comparison results of contact angles on different samples.

2.5. Statistical Analysis. All the data were expressed as the means \pm standard deviation $(N=3)$ for each experiment. The level of significance was determined by the analysis of variance, followed by Student-Newman-Keuls post hoc test for multiple comparisons. $p<0.05$ was considered significant, and $p<0.01$ was considered highly significant. Significance levels were ${ }^{*} p<0.05$ and ${ }^{* *} p<0.01$ when compared with $\mathrm{Ti}$, ${ }^{\#} p<0.05$ and ${ }^{\# \#} p<0.01$ when compared with NT10, and $\otimes_{p}<0.05$ and ${ }^{\& \&} p<0.01$ when compared with NT30.

\section{Results}

\subsection{Characterization of Specimens}

3.1.1. SEM. As shown by the SEM images in Figure 1, pure Ti possessed random microrough surface features at low magnification and lacked evident nanotubes at high magnification. By contrast, NT10, NT30, and NT60 showed groove-like topography at low magnification and highly ordered nanotubes with diameters of approximately 30,100 , and $200 \mathrm{~nm}$, respectively, at high magnification, just as shown in our previous work [13], thereby resulting in a dual micro- and nanorough surface.

3.1.2. XRD. XRD patterns (Figure 2) showed that all three TNTs featured Ti and anatase peaks without any rutile nor amorphous peaks after annealing in air at $450^{\circ} \mathrm{C}$ for $2 \mathrm{~h}$. Compared with the rutile and amorphous crystal types, the anatase crystal type possessed good antibacterial property 

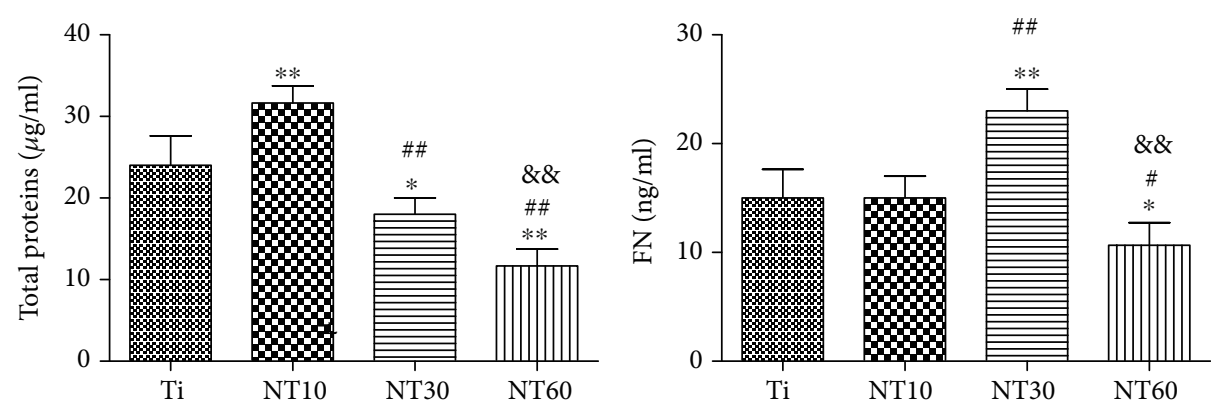

FIgURE 5: Absorbed total proteins and FN after $2 \mathrm{~h}$ of immersion in medium containing 10\% FBS.

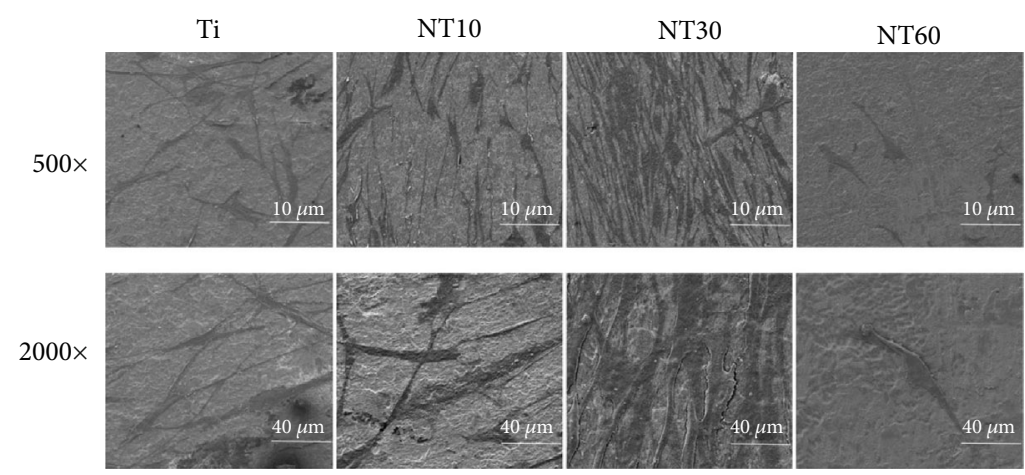

FIGURE 6: SEM images with two different magnifications of 500x and 2,000x showing the morphologies of HGFs after 2 days of culture on specimens.

and may improve the biocompatibility by favoring protein adhesion $[20,21]$.

3.1.3. AFM. The topographical features were evaluated by AFM (Figure 3). The prepared TNTs possessed a mechanically prepared groove-like topography, whereas the pure $\mathrm{Ti}$ featured a random micron-size rough topography. The roughness values of the samples decreased in the following order: Ti $>$ NT10 $>$ NT60 > NT30.

3.1.4. Static Contact Angles. The wettability of samples was detected by measuring the water contact angles (Figure 4). The TNTs showed favorable hydrophilia with a relatively low water contact angle, and the hydrophilicity increased with the increase in diameter. By contrast, pure Ti was hydrophobic.

3.1.5. Total Proteins and FN Adsorption Assay. Figure 5 shows the amounts of adsorbed total proteins and FN from $10 \%$ FBS after $2 \mathrm{~h}$ of immersion. Compared with Ti, NT10 absorbed more total proteins whereas NT30 and NT60 absorbed less amount of proteins. NT30 absorbed more FN than Ti and NT10. Meanwhile, NT60 absorbed the least amount of FN.

\subsection{In Vitro Biocompatibility Studies}

3.2.1. Cell Morphology. The HGFs cultured on different surfaces exhibited noticeably varying shapes (Figure 6). Most of the HGFs on control Ti exhibited an oval shape without the noticeable filopodia extensions and had an irregular arrangement. The cells on NT10 and NT30 elongated further and featured a large number of prominent filopodia and lamellipodia extensions and were arranged orderly along the direction of the grooves, especially on NT30. The cells on NT60 were sparsely distributed and retained their slender shape without noticeable filopodia extensions.

3.2.2. Adhesion and Proliferation. The initial adherent cell number was surveyed using DAPI staining (Figure 7). Cell attachment was significantly improved on NT10 and NT30 but was severely inhibited on NT60. Cell proliferation was surveyed using the CCK-8 assay (Figure 8). Cell proliferation on all surfaces increased with time. NT10 and NT30 could promote cell proliferation when compared with control $\mathrm{Ti}$, with the latter showing a stronger promotion, although no statistically significant difference was found between $\mathrm{Ti}$ and NT10 on day 7. By contrast, cell attachment was severely inhibited on NT60.

3.2.3. Gene Expressions. The expression levels of ECMrelated genes, including those of ITGB1, VCL, FN, and COL1, were assessed by qRT-PCR (Figure 9). The expression levels of relevant genes became increasingly higher with the increased diameter of NT10 and NT30, although no significant difference in the expressions of ITGB and COL1 was observed between NT10 and control Ti. However, the expression levels of ECM-related genes were downregulated on NT60. 

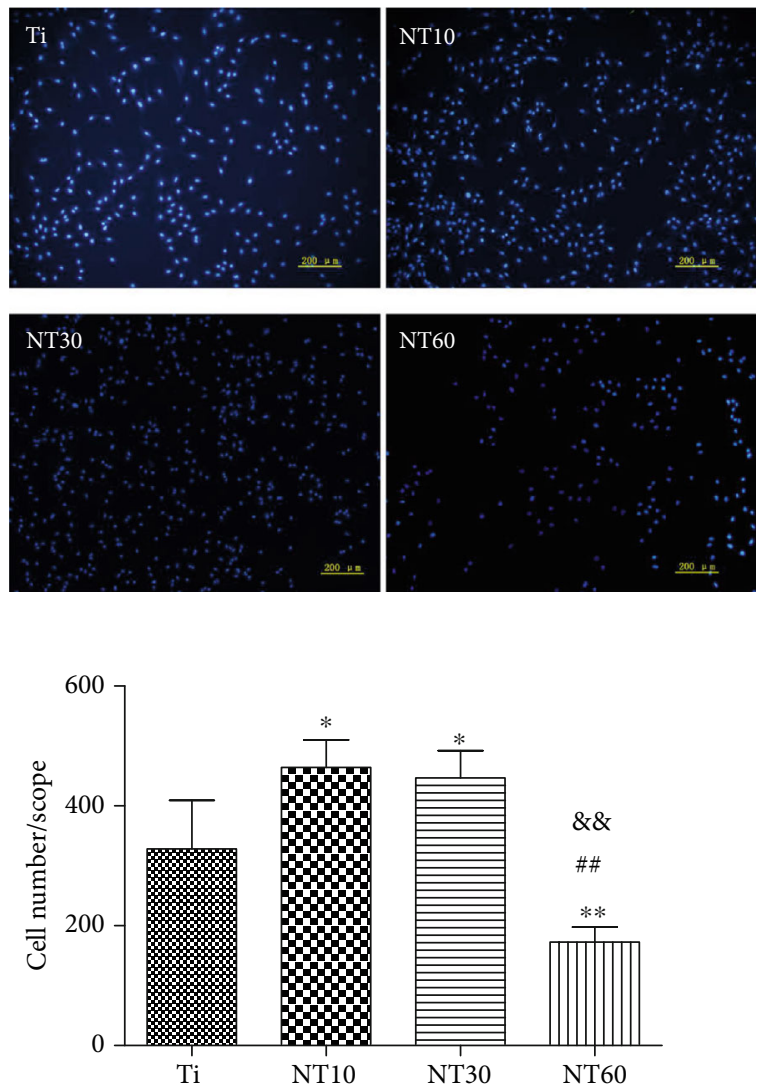

FIGURE 7: Fluorescence images of initial adherent HGFs stained with DAPI and cell numbers measured by counting cells after $2 \mathrm{~h}$.

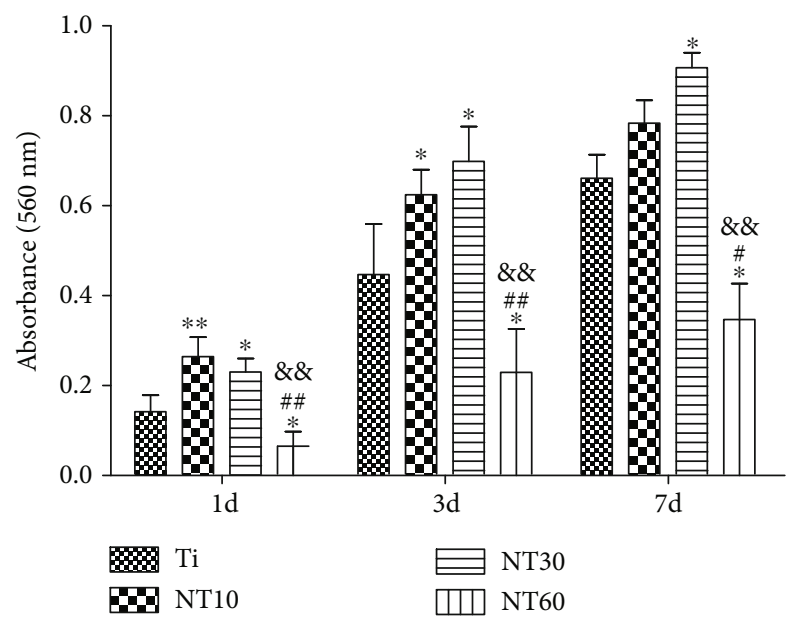

Figure 8: Cell proliferation measured by CCK-8 assay after culturing HGFs on different samples for 1,3 , and 7 days.

3.3. Antibacterial Activity. As shown in Figure 10, P. gingivalis displayed their normal rod shapes on control Ti and partly shrunk to round shapes and were distributed sparsely on TNTs, especially on NT30 and NT60. The number of viable bacteria on the samples decreased in the following order: $\mathrm{Ti}$ $>$ NT10 > NT60 > NT30.

\section{Discussions}

The successful long-term function of a dental implant relies not only on the ideal osseointegration but also on the effective interaction of the implant with the surrounding soft tissues [22]. Soft-tissue integration with the dental implant establishes a biological seal between the oral environment and the implant. This characteristic inhibits unfavorable soft tissue recession and marginal bone resorption and contributes to the resistance against bacterial invasion [17]. However, although many studies have focused on the osseointegration of the body surface, few studies have been conducted for the design of transgingival surface. Many studies have recently shown that a microrough surface could establish a more stable soft-tissue attachment compared with the traditionally smooth surface and is thus more suitable for the transgingival part of dental implant [23]. In this work, TNTs with three different diameters were manufactured on the microrough $\mathrm{Ti}$ sheet, and the microrough $\mathrm{Ti}$ sheet with a native $\mathrm{TiO}_{2}$ oxide layer was used as the control. We previously confirmed that TNTs with a diameter of $100 \mathrm{~nm}$ could increase the response of mesenchymal stem cells and simultaneously decrease the adhesion of $S$. aureus [13]. Thus, in this study, we fully investigated the biological behavior of HGFs and the adhesion of $P$. gingivalis, which determined the peri-implant soft-tissue integration.

Upon contact of an implant surface with blood, plasma proteins are spontaneously adsorbed onto the implant surface within seconds and convey the effect of topographical cues to the attached cells/tissues to direct the biological performance of biomaterials [24, 25]. NT10 (diameter: $30 \mathrm{~nm}$ ) with higher hydrophilicity induced more total proteins and thus improved the cell attachment at $2 \mathrm{~h}$ compared with $\mathrm{Ti}$. The total protein aggregates $(\approx 30 \mathrm{~nm}$-size regime) are too small to anchor on NT30 (diameter: $100 \mathrm{~nm}$ ) and NT60 (diameter: $200 \mathrm{~nm}$ ), although both are more hydrophilic than $\mathrm{Ti}[13,25]$. However, plasma FN ( $\approx 120 \mathrm{~nm}$-size regime) [26, 27], which plays an important role in the adhesion of HGFs onto the implant surface, could be more easily adsorbed onto NT30 than onto the other samples (Figure 4). Thus, NT30 induced more cell attachments than the other samples possibly because of the increased FN adhesions. However, NT60 induced less amounts of total proteins and $\mathrm{FN}$ and thus less achieved cell attachment than Ti. Furthermore, the TNTs showed lower roughness values than $\mathrm{Ti}$, with NT30 achieving the lowest value. Fibroblasts are sensitive not only to the changes in hydrophilicity but also to those in Sa which influences the assembly of focal adhesion (FA) and subsequent intracellular signaling cascade activation $[28,29]$. Only the roughness of NT30 was less than $0.5 \mu \mathrm{m}$ (Figure 3), whereas the surface with a roughness less than $0.5 \mu \mathrm{m}$ was considered to feature a smooth surface [30]. This finding is consistent with the conclusion that highly ordered and smooth TNTs could be fabricated by applying a suitable range of voltage, and the TNTs would become extremely compact or spongy-like and porous if the applied potential voltage was notably low or extremely high [31]. Given its low roughness, NT30 in this study might benefit the attachment of HGFs, because HGFs tend to adhere to smooth surfaces [32, 33]. 


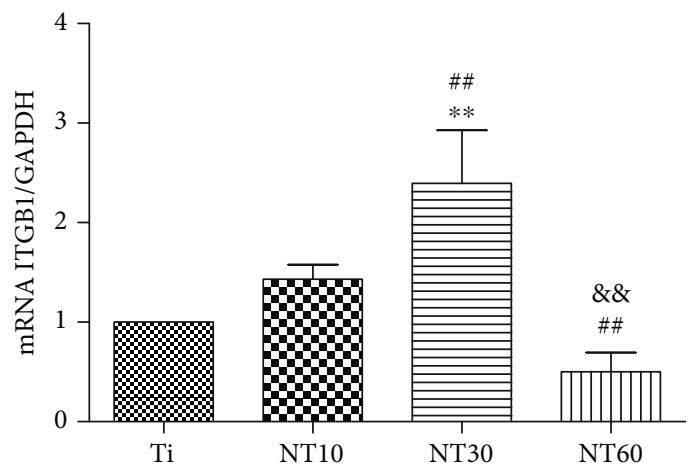

(a)

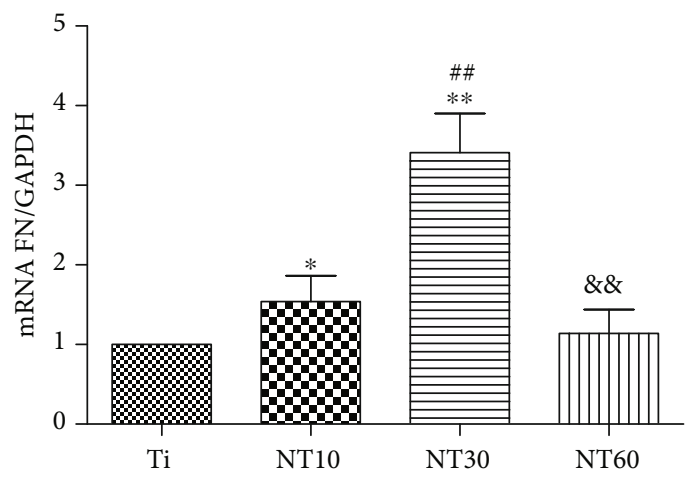

(c)

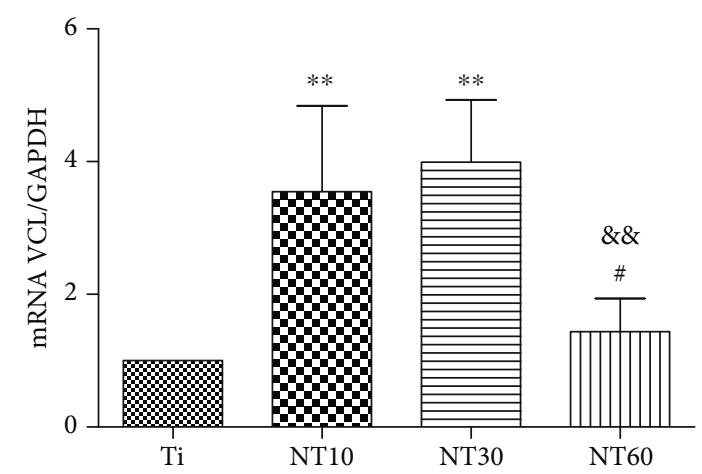

(b)

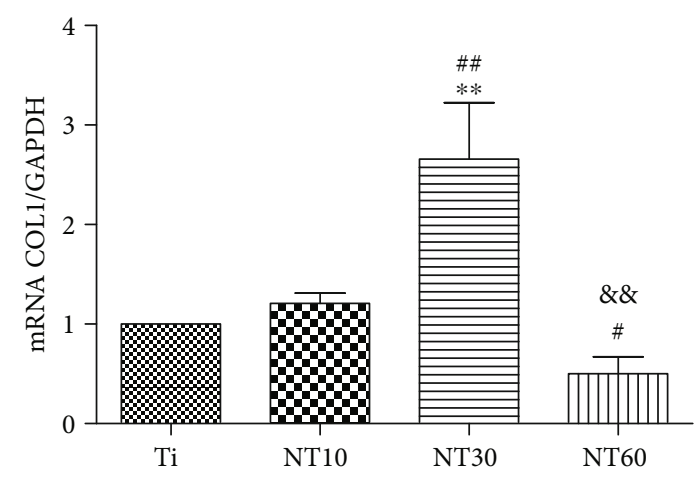

(d)

Figure 9: Relative expressions of (a) ITGB1, (b) VCL, (c) FN, and (d) COL1 by HGFs seeding on different substrates for 7 days.

The cell proliferation assay results showed that NT10 and NT30 could promote the cell proliferation compared with control Ti, with NT30 showing the stronger promotion. This finding is due to not only additional cell adhesions but also the increased surface area available for cell colonization and increased fluid exchange. The proliferation on NT60 was significantly inhibited, because extremely low protein amounts and cell adhesion could potentially lead to cell quiescence and apoptosis (oval-shaped HGFs without noticeable extensions on NT60 as shown in Figure 6, indicating cell death or cells with low activity).

HGFs cultured on NT10 and NT30 extended across the tubes to reach a protein-deposited surface for initial contact, thus further elongating and showing a large number of prominent filopodia and lamellipodia extensions according to the SEM images (Figure 6). An extensively stretched appearance indicated evidence of migration and activation [34]. The cells were also aligned in the same direction, which could be related to the underlying micro-/nanorough groovelike topographical features $[35,36]$. The anisotropic groovelike surface texture created mechanical stress, which directed the cell fate via contact guidance, i.e., the attachment, proliferation, spread, migration, and alignment of cells [37]. Cell alignment plays a critical role in various cell behaviors, including membrane protein relocation, cytoskeleton reorganization, related gene expression, and ECM remodeling [14, 38]. It has been demonstrated that HGFs attach to the material surface through FAs, which anchor the cells to the substratum and can mediate both mechanical and biochemical signaling [39]. Thus, we investigated the expression levels of ECM-related genes, including those of ITGB, VCL, FN, and COL1. Our results showed that NT10 and NT30 significantly promoted the expressions of ECM-related genes and demonstrated excellent activity, with the latter exhibiting a higher promotion. HGFs on NT60 retained their oval shape without noticeable filopodia extension, indicating cell death or cells with a low activity, and thus the lack of noticeable related gene expressions. This finding is in accordance with the discovery that fibroblasts respond to surface topography by changes in the alignment and subsequent ECM production $[9,35]$. FN, which occurs in two forms, namely, the soluble plasma FN in blood and insoluble cellular FN in ECM or on cell surfaces, is a key factor in the compatibility of dental implant materials for connective tissues [40]. VCL is a FA linker protein, and increased VCL expression is associated with enhanced adhesion strength [41]. The high expressions of cellular FN and VCL might also facilitate the adhesion of HGFs on NT10 and NT30. Furthermore, the groove-like surface texture on NT10 and NT30 could also contribute to promote more adhesion and proliferation via contact guidance.

The early failure of dental implants mostly results from bacteria and plaque formation on the implant surfaces before soft-tissue integration occurs, especially in the cases of periodontal disease [42]. Given the difficulty of killing bacteria after binding to the implant surface, prevention strategies have therefore become extremely important [43]. In our previous study, TNTs exhibited higher hydrophilicity and bioactivity in the anatase-phase after heat treatment, thereby decreasing the adhesion of S. aureus [13]. In this work, TNTs demonstrated the same characteristics by contact angle 

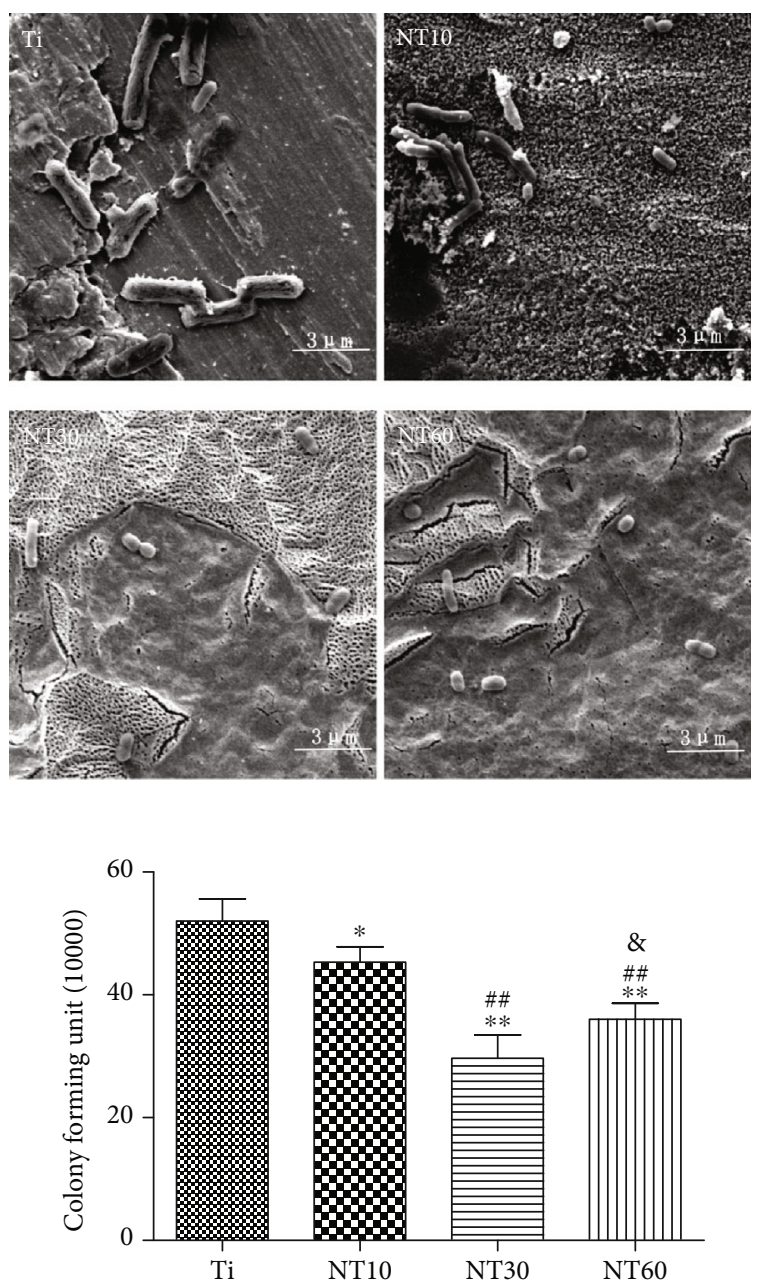

FIGURE 10: SEM images and quantitative analysis of viable adherent $P$. gingivalis on the samples after $6 \mathrm{~h}$.

determination and phase analysis, thus reducing bacterial attachment. The bacteria shrunk to round shapes and were distributed sparsely, indicating their bacteria inactivation. This finding is consistent with our previous work. NT30 achieved the highest reduction of bacterial adhesion in this work, whereas in our previous research, this finding was observed with NT60. This finding is probably due to the use of Gram-negative P. gingivalis in the present work, whereas the Gram-positive S. aureus was used in our previous research. Surface properties, including roughness, hydrophilicity, and chemical composition, all affect the adhesion of bacteria [44]. However, the initial bacterial adhesion, especially that of Gram-negative bacteria with a thinner cell wall, is more easily influenced by roughness than by hydrophilicity [45]. Thus, NT30 caused the most reduction in the adhesion of $P$. gingivalis possibly because of its roughness $(\mathrm{Sa}<0.5 \mu \mathrm{m}$ ), which was the lowest detected by AFM. The obtained results are consistent with those obtained by a previous study, in which the smooth surface of machined neck $(\mathrm{Sa}<0.5 \mu \mathrm{m}$ ) was generally conducive for the attachment of soft tissue while contributing to the inhibition of bacterial biofilm adhesion [46].

\section{Conclusion}

The amount and bioactivity of HGFs existing in the implantmucosa interface are important in the formation of softtissue seals, which could block bacteria from entering the space between the transgingival part and the biological tissue $[17,47]$. NT30 could bioactivate HGF functions to the utmost, including not only cell attachment and proliferation but also ECM-related gene expressions. Bioactivating HGFs could increase the possibility to win the "race for the surface" against bacteria at the attachment sites. Moreover, NT30 could also reduce the adhesion of $P$. gingivalis to the transgingival part, facilitating the attachment of soft tissue, inhibiting the adhesion of bacterial biofilm, and preserving the crestal bone. Findings from previous and current studies indicate that NT30 might feature considerable potential in the intraosseous and transgingival parts of dental implants. Further studies are needed to investigate the in vivo and clinical implications when the results are applied for clinical use.

\section{Data Availability}

The data used to support the finding of this study are included within the article.

\section{Conflicts of Interest}

The authors declare no conflict of interest.

\section{Acknowledgments}

This work is supported by Fujian Health and Family Planning Young and Middle-aged Talents Training Project (2017-ZQN-79) and Putian Science and Technology Project (2018S3F007).

\section{References}

[1] S. Roehling, K. A. Schlegel, H. Woelfler, and M. Gahlert, "Zirconia compared to titanium dental implants in preclinical studies-a systematic review and meta-analysis," Clinical Oral Implants Research, vol. 30, no. 5, pp. 365-395, 2019.

[2] A. Messias, P. Nicolau, and F. Guerra, "Titanium dental implants with different collar design and surface modifications: a systematic review on survival rates and marginal bone levels," Clinical Oral Implants Research, vol. 30, no. 1, pp. 2048, 2019.

[3] S. Mei, H. Wang, W. Wang et al., "Antibacterial effects and biocompatibility of titanium surfaces with graded silver incorporation in titania nanotubes," Biomaterials, vol. 35 , no. 14 , pp. 4255-4265, 2014.

[4] X. Liu, X. Zhou, S. Li et al., "Effects of titania nanotubes with or without bovine serum albumin loaded on human gingival fibroblasts," International Journal of Nanomedicine, vol. 9, p. 1185, 2014.

[5] D. A. Moin, B. Hassan, P. Mercelis, and D. Wismeijer, "Designing a novel dental root analogue implant using cone beam computed tomography and CAD/CAM technology," Clinical Oral Implants Research, vol. 24, pp. 25-27, 2013.

[6] C. Pierre, G. Bertrand, C. Rey, O. Benhamou, and C. Combes, "Calcium phosphate coatings elaborated by the soaking 
process on titanium dental implants: Surface preparation, processing and physical-chemical characterization," Dental Materials, vol. 35, no. 2, pp. e25-e35, 2019.

[7] M. Ferrà-Cañellas, M. Llopis-Grimalt, M. Monjo, and J. Ramis, "Tuning nanopore diameter of titanium surfaces to improve human gingival fibroblast response," International Journal of Molecular Sciences, vol. 19, no. 10, p. 2881, 2018.

[8] R. Xu, X. Hu, X. Yu et al., "Micro-/nano-topography of selective laser melting titanium enhances adhesion and proliferation and regulates adhesion-related gene expressions of human gingival fibroblasts and human gingival epithelial cells," International Journal of Nanomedicine, vol. Volume 13, pp. 5045-5057, 2018.

[9] K. Gulati, H.-J. Moon, T. Li, P. T. Sudheesh Kumar, and S. Ivanovski, "Titania nanopores with dual micro-/nanotopography for selective cellular bioactivity," Materials Science and Engineering: $C$, vol. 91, pp. 624-630, 2018.

[10] H. Wei, Feng, Wu et al., "Increased fibroblast functionality on CNN2-loaded titania nanotubes," International Journal of Nanomedicine, vol. 7, p. 1091, 2012.

[11] H. C. Li, Y. M. Zhang, and H. P. Sun, "Effect of diametercontrolled $\mathrm{Ti}-\mathrm{TiO} 2$ nanotubes on the adhesion of osteoblast and fibroblast," Chinese journal of stomatology, vol. 47, pp. 122-126, 2012.

[12] L. Zhou, Y. Lai, W. Huang et al., "Biofunctionalization of microgroove titanium surfaces with an antimicrobial peptide to enhance their bactericidal activity and cytocompatibility," Colloids and Surfaces B: Biointerfaces, vol. 128, pp. 552-560, 2015.

[13] Z. Xu, Y. Lai, D. Wu et al., "Increased mesenchymal stem cell response and decreased staphylococcus aureus adhesion on titania nanotubes without pharmaceuticals," BioMed Research International, vol. 2015, 9 pages, 2015.

[14] Y. Lai, J. Chen, T. Zhang et al., "Effect of 3D microgroove surface topography on plasma and cellular fibronectin of human gingival fibroblasts," Journal of Dentistry, vol. 41, no. 11, pp. 1109-1121, 2013.

[15] P. R. Kramer, A. JanikKeith, Z. Cai, S. Ma, and I. Watanabe, "Integrin mediated attachment of periodontal ligament to titanium surfaces," Dental Materials, vol. 25, no. 7, pp. 877-883, 2009.

[16] B. Steffensen, L. Häkkinen, and H. Larjava, "Proteolytic events of wound-healing-coordinated interactions among matrix metalloproteinases (MMPs), integrins, and extracellular matrix molecules," Critical Reviews in Oral Biology \& Medicine, vol. 12, no. 5, pp. 373-398, 2016.

[17] X. Wang, T. Lu, J. Wen et al., "Selective responses of human gingival fibroblasts and bacteria on carbon fiber reinforced polyetheretherketone with multilevel nanostructured $\mathrm{TiO} 2$," Biomaterials, vol. 83, pp. 207-218, 2016.

[18] T. Rozario and D. W. DeSimone, "The extracellular matrix in development and morphogenesis: a dynamic view," Developmental Biology, vol. 341, no. 1, pp. 126-140, 2010.

[19] Q. Ma, S. Mei, K. Ji, Y. Zhang, and P. K. Chu, "Immobilization of Ag nanoparticles/FGF-2 on a modified titanium implant surface and improved human gingival fibroblasts behavior," Journal of Biomedical Materials Research Part A, vol. 98, no. 2, pp. 274-286, 2011.

[20] K. S. Brammer, S. Oh, C. J. Cobb, L. M. Bjursten, H. v. d. Heyde, and S. Jin, "Improved bone-forming functionality on diameter-controlled $\mathrm{TiO}_{2}$ nanotube surface," Acta Biomaterialia, vol. 5, no. 8, pp. 3215-3223, 2009.

[21] O. Zinger, K. Anselme, A. Denzer et al., "Time-dependent morphology and adhesion of osteoblastic cells on titanium model surfaces featuring scale-resolved topography," Biomaterials, vol. 25, no. 14, pp. 2695-2711, 2004.

[22] Y. Wang, Y. Zhang, and R. J. Miron, "Health, maintenance, and recovery of soft tissues around implants," Clinical Implant Dentistry and Related Research, vol. 18, no. 3, pp. 618-634, 2016.

[23] L. Canullo, M. Annunziata, P. Pesce, G. Tommasato, L. Nastri, and L. Guida, "Influence of abutment material and modifications on peri-implant soft-tissue attachment: a systematic review and meta-analysis of histological animal studies," The Journal of Prosthetic Dentistry, 2020.

[24] M. S. Lord, M. Foss, and F. Besenbacher, "Influence of nanoscale surface topography on protein adsorption and cellular response," Nano Today, vol. 5, no. 1, pp. 66-78, 2010.

[25] S. Oh, K. S. Brammer, Y. S. J. Li et al., "Stem cell fate dictated solely by altered nanotube dimension," Proceedings of the National Academy of Sciences, vol. 106, no. 7, pp. 2130-2135, 2009.

[26] H. P. Erickson and N. A. Carrell, "Fibronectin in extended and compact conformations. Electron microscopy and sedimentation analysis," Journal of Biological Chemistry, vol. 258, pp. 14539-14544, 1984.

[27] E. Gongadze, D. Kabaso, S. Bauer, J. Park, P. Schmuki, and A. Iglic, "Adhesion of osteoblasts to a vertically aligned $\mathrm{TiO} 2$ nanotube surface," Mini Reviews in Medicinal Chemistry, vol. 13, no. 2, pp. 194-200, 2013.

[28] C. J. Oates, W. Wen, and D. W. Hamilton, "Role of titanium surface topography and surface wettability on focal adhesion kinase mediated signaling in fibroblasts," Materials, vol. 4, no. 5, pp. 893-907, 2011.

[29] C. Zhou, F. Lei, J. Chodosh, and E. I. Paschalis, "The role of titanium surface microtopography on adhesion, proliferation, transformation, and matrix deposition of corneal cells," Investigative Ophthalmology \& Visual Science, vol. 57, no. 4, p. 1927, 2016.

[30] T. Albrektsson and A. Wennerberg, "Oral implant surfaces: Part 1-review focusing on topographic and chemical properties of different surfaces and in vivo responses to them," International Journal of Prosthodontics, vol. 17, pp. 536-543, 2004.

[31] Y. Fu and A. Mo, "A review on the electrochemically selforganized titania nanotube arrays: synthesis, modifications, and biomedical applications," Nanoscale Research Letters, vol. 13, no. 1, p. 187, 2018.

[32] J. Cao, T. Wang, Y. Pu, Z. Tang, and H. Meng, "Influence on proliferation and adhesion of human gingival fibroblasts from different titanium surface decontamination treatments: an in vitro study," Archives of Oral Biology, vol. 87, pp. 204-210, 2018.

[33] D. M. Brunette, "Principles of cell behavior on titanium surfaces and their application to implanted devices," in In Titanium in medicine, pp. 485-512, Springer, 2001.

[34] L. Fathyunes, J. Khalil-Allafi, S. O. R. Sheykholeslami, and M. Moosavifar, "Biocompatibility assessment of graphene oxide-hydroxyapatite coating applied on $\mathrm{TiO}_{2}$ nanotubes by ultrasound-assisted pulse electrodeposition," Materials Science \& Engineering. C, Materials for Biological Applications, vol. 87, pp. 10-21, 2018. 
[35] S. Ferraris, F. Truffa Giachet, M. Miola et al., "Nanogrooves and keratin nanofibers on titanium surfaces aimed at driving gingival fibroblasts alignment and proliferation without increasing bacterial adhesion," Materials Science and Engineering: $C$, vol. 76, pp. 1-12, 2017.

[36] P. Zhou, F. Mao, F. He et al., "Screening the optimal hierarchical micro/nano pattern design for the neck and body surface of titanium implants," Colloids and Surfaces B: Biointerfaces, vol. 178, pp. 515-524, 2019.

[37] X. F. Walboomers, L. A. Ginsel, and J. A. Jansen, "Early spreading events of fibroblasts on microgrooved substrates," Journal of Biomedical Materials Research Part B Applied Biomaterials, vol. 51, no. 3, pp. 529-534, 2000.

[38] Y. Li, G. Huang, X. Zhang et al., "Engineering cell alignment in vitro," Biotechnology Advances, vol. 32, no. 2, pp. 347-365, 2014.

[39] C. J. Pendegrass, C. A. Middleton, D. Gordon, J. Jacob, and G. W. Blunn, "Measuring the strength of dermal fibroblast attachment to functionalized titanium alloys in vitro," Journal of Biomedical Materials Research Part A: An Official Journal of The Society for Biomaterials, The Japanese Society for Biomaterials, and The Australian Society for Biomaterials and the Korean Society for Biomaterials, vol. 92, pp. 1028-1037, 2010.

[40] Wing S To and K. S. Midwood, "Plasma and cellular fibronectin: distinct and independent functions during tissue repair," Fibrogenesis \& Tissue Repair, vol. 4, no. 1, 2011.

[41] Y.-H. Moon, M.-K. Yoon, J.-S. Moon et al., "Focal adhesion linker proteins expression of fibroblast related to adhesion in response to different transmucosal abutment surfaces," The journal of advanced prosthodontics, vol. 5, no. 3, pp. 341-350, 2013.

[42] E. D. de Avila, B. A. Oirschot, and J. J. J. P. van den Beucken, "Biomaterial-based possibilities for managing peri-implantitis," Journal of Periodontal Research, vol. 55, no. 2, pp. 165173, 2020.

[43] C. A. Fux, J. W. Costerton, P. S. Stewart, and P. Stoodley, "Survival strategies of infectious biofilms," Trends in Microbiology, vol. 13, no. 1, pp. 34-40, 2005.

[44] M. Yoshinari, Y. Oda, T. Kato, K. Okuda, and A. Hirayama, "Influence of surface modifications to titanium on oral bacterial adhesionin vitro," Journal of Biomedical Materials Research Part A, vol. 52, no. 2, pp. 388-394, 2000.

[45] A. Pier-Francesco, R. J. Adams, M. G. J. Waters, and D. W. Williams, "Titanium surface modification and its effect on the adherence of Porphyromonas gingivalis: an in vitro study," Clinical Oral Implants Research, vol. 17, no. 6, pp. 633-637, 2006.

[46] M. Köunönen, M. Hormia, J. Kivilahti, J. Hautaniemi, and I. Thesleff, "Effect of surface processing on the attachment, orientation, and proliferation of human gingival fibroblasts on titanium," Journal of Biomedical Materials Research, vol. 26, no. 10, pp. 1325-1341, 1992.

[47] C. Lavelle, "Mucosal seal around endosseous dental implants," The Journal of Oral Implantology, vol. 9, no. 3, pp. 357-371, 1981. 